\title{
Effects of Surface Morphology on the Optical and Electrical properties of Schottky diodes of CBD deposited ZnO Nanostructures
}

Benard S. Mwankemwa ${ }^{1,2^{*}}$, Shadrach Akinkuade ${ }^{1}$, Kelebogile Maabong ${ }^{1,3}$, Jackie M. Nel ${ }^{1}$ and Mmantsae Diale ${ }^{1}$

\footnotetext{
${ }^{1}$ Department of Physics, University of Pretoria, Private bag X20, Hatfield, Republic of South Africa.

${ }^{2}$ Department of Physics, School of Physical Sciences, College of Natural and Mathematical

Sciences, University of Dodoma, P. O. Box 338, Dodoma, Tanzania.

${ }^{3}$ Department of Physics, University of Botswana, Private Bag 0022 Gaborone, Botswana

*Corresponding author: benard_80@yahoo.com
}

\begin{abstract}
.
We report on effect of surface morphology on the optical and electrical properties of chemical bath deposited Zinc oxide $(\mathrm{ZnO})$ nanostructures. $\mathrm{ZnO}$ nanostructures were deposited on the seeded conducting indium doped tin oxide substrate positioned in three different directions in the growth solution. Field emission scanning electron microscopy was used to evaluate the morphological properties of the synthesized nanostructures and revealed that the positioning of the substrate in the growth solution affects the surface morphology of the nanostructures. The optical absorbance, photoluminescence and Raman spectroscopy of the resulting nanostructures are discussed. The electrical characterization of the Schottky diode such as barrier height, ideality factor, rectification ratios, reverse saturation current and series resistance were found to depend on the nanostructures morphology. In addition, current transport mechanism in the higher forward bias of the Schottky diode was studied and space charge limited current was found to be the dominant transport mechanism in all samples.
\end{abstract}

Keywords: $\mathrm{ZnO}$ nanostructures, Surface morphology, Optical properties, Electrical characterization, Transport mechanism 


\section{Introduction}

Zinc oxide $(\mathrm{ZnO})$ is the most successful metal oxide semiconductor in its nanostructure form due to its numerous applications in optoelectronic devices [1,2]. Many of these potential applications of $\mathrm{ZnO}$ are due to the reasons that, it has a wide direct band gap of $3.37 \mathrm{eV}$ and prominent feature of having high excitonic energy of $60 \mathrm{meV}$. Different morphologies of $\mathrm{ZnO}$ nanostructures have been synthesized and used in different applications [3-5]. It is clear that the morphology of $\mathrm{ZnO}$ nanostructures including shape, size, density and orientation plays a crucial role in the performance of devices under investigation. A study by Zhiqiang et al [6] proved that the morphology of the $\mathrm{ZnO}$ as a buffer layer plays a critical role in photovoltaic properties of inverted polymer solar cells. Oh et al [7] demonstrated improved photon extraction efficiency as a result different morphologies attained by modulating $\mathrm{ZnO}$ using organic acids.

Herein we report the effect of surface morphology on the optical and electrical properties of Schottky diodes based on $\mathrm{ZnO}$ nanostructures. The nanostructures were synthesized using a chemical bath deposition (CBD) method without addition of surfactants/catalysts. Three different morphologies were achieved by changing the orientation of the substrates in the growth solution.

\section{Materials and Experimental Procedures}

$\mathrm{ZnO}$ nanostructures have been synthesized on $2 \times 1 \mathrm{~cm}^{2}$ ITO coated substrates by seed-layer assisted CBD. Prior to deposition, the substrates were cleaned by sonicating in deionized water, acetone, ethanol for $5 \mathrm{~min}$ each, and then dried using $\mathrm{N}_{2}$ gas. The detailed procedures for the preparation of the gel, deposition of the seed layer and growth of $\mathrm{ZnO}$ nanorods are as described in our previous work [8]. However, in this study the nanostructure growth was achieved by suspending substrates with the seeded surface facing: downward (Sample 1), 
vertically (Sample 2) and up (Sample 3) as shown in Fig 1 (a). The beaker with growth solution was placed in a water bath maintained at $90^{\circ} \mathrm{C}$ for 2 hours and under constant stirring throughout the experiment. The obtained $\mathrm{ZnO}$ nanostructures were rinsed with $\mathrm{DI}$ water to remove loose particles on the surface and dried using $\mathrm{N}_{2}$ gas and all characterizations were done on the as-synthesized nanostructures (no post-treatment were done). The surface morphology of the as-synthesized samples was studied using field emission scanning electron microscopy (FE - SEM, ZEISS SEM-Microscope Crossbeam540). X-ray diffraction (XRD) was used to determine the crystalline quality of the samples using XPERT-PRO diffractometer in a $\theta / 2 \theta$ configuration (PANalytical BV, Netherlands). Optical absorbance of the nanostructures was measured using a Varian Cary 100 UV-Vis Spectrophotometer. The baseline correction was performed first by placing two ITO substrates with the coated side facing the light sources. Thereafter the substrate with $\mathrm{ZnO}$ nanostructures facing the light source was placed firmly in one of the sample holders (while the other having ITO substrate). The absorbance measurements were carried out in the wavelength ranging from $200 \mathrm{~nm}$ to $800 \mathrm{~nm}$. Room temperature (RT) photoluminescence (PL) measurements were done using a Varian Cary Eclipse EL04103870 fluorescence spectrophotometer at an excitation wavelength of $325 \mathrm{~nm}$. Raman studies were performed using Raman spectrometer Jobin Yvon, Horiba $^{(\mathrm{C})}$ TX64000. Resistive evaporation unit was then used to deposit $1000 \AA$ gold (Au) metal at a pressure of $4 \times 10^{-6}$ mbar using shadow mask to form $\mathrm{Au} / \mathrm{ZnO} / \mathrm{ITO}$ Schottky diodes. In this arrangement, Au was used as Schottky contacts while ohmic contact was achieved using ITO. The current-voltage $(I-V)$ properties of the Schottky diode were measured at RT using HP 4140 B pA Meter DC voltage Source. 


\section{Results and discussion}

\subsection{Surface Morphology}

The SEM micrographs presented in Fig. 1 (b) - (d) shows three different morphologies of synthesized $\mathrm{ZnO}$ nanostructures. The orientation of the seeded substrates has a major influence on the density and shape of the synthesized nanostructures. Vertically arranged nanorods with well-defined hexagonal flat tops were observed on the seeded substrate placed facing down Fig. 1(b). For the substrate that was placed vertically the narrower rod-like nanostructures with reduced size grew parallel to the substrate, Fig. 1 (c). Fig. 1 (d) shows nanostructures obtained on face up seeded substrate. Here a remarkable change in the morphology occurred and densely agglomerated, small nanoparticles were obtained. This might be due to the precipitates that settled on top of the substrate, resulting in both primary and secondary growth favoring multidirectional growth. However, growth mechanism for the growth of different structures of $\mathrm{ZnO}$ have been described elsewhere [9].

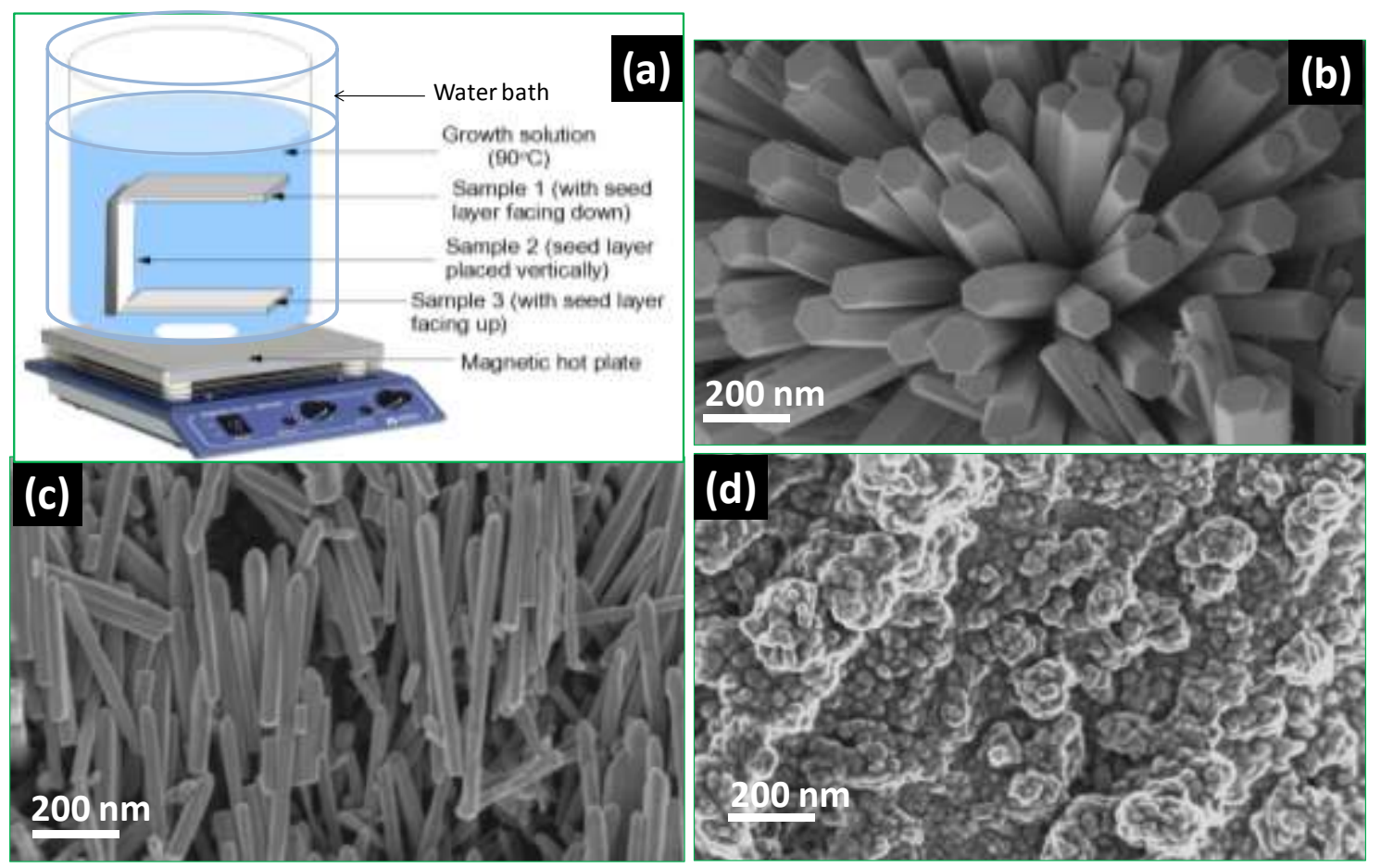

Fig. 1. (a) Experimental setup showing substrate orientations. SEM images of ZnO nanostructures: (a) hexagonal shaped nanorods, Sample 1 (b) narrower rod-like structures, Sample 2 and (c) agglomerated small nanoparticles, Sample 3. 


\subsection{Structural properties}

Microstructural analyses of the synthesized nanostructures are as displayed in Fig. 2. All major sharp peaks in the range $2 \theta \sim 20^{\circ}-70^{\circ}$ of all the nanostructures studied were indexed to the hexagonal wurtzite structure of $\mathrm{ZnO}$ (JCPDS file number 36-1451). No characteristic peaks of other new phases were observed indicating the purity of the synthesized nanostructures.

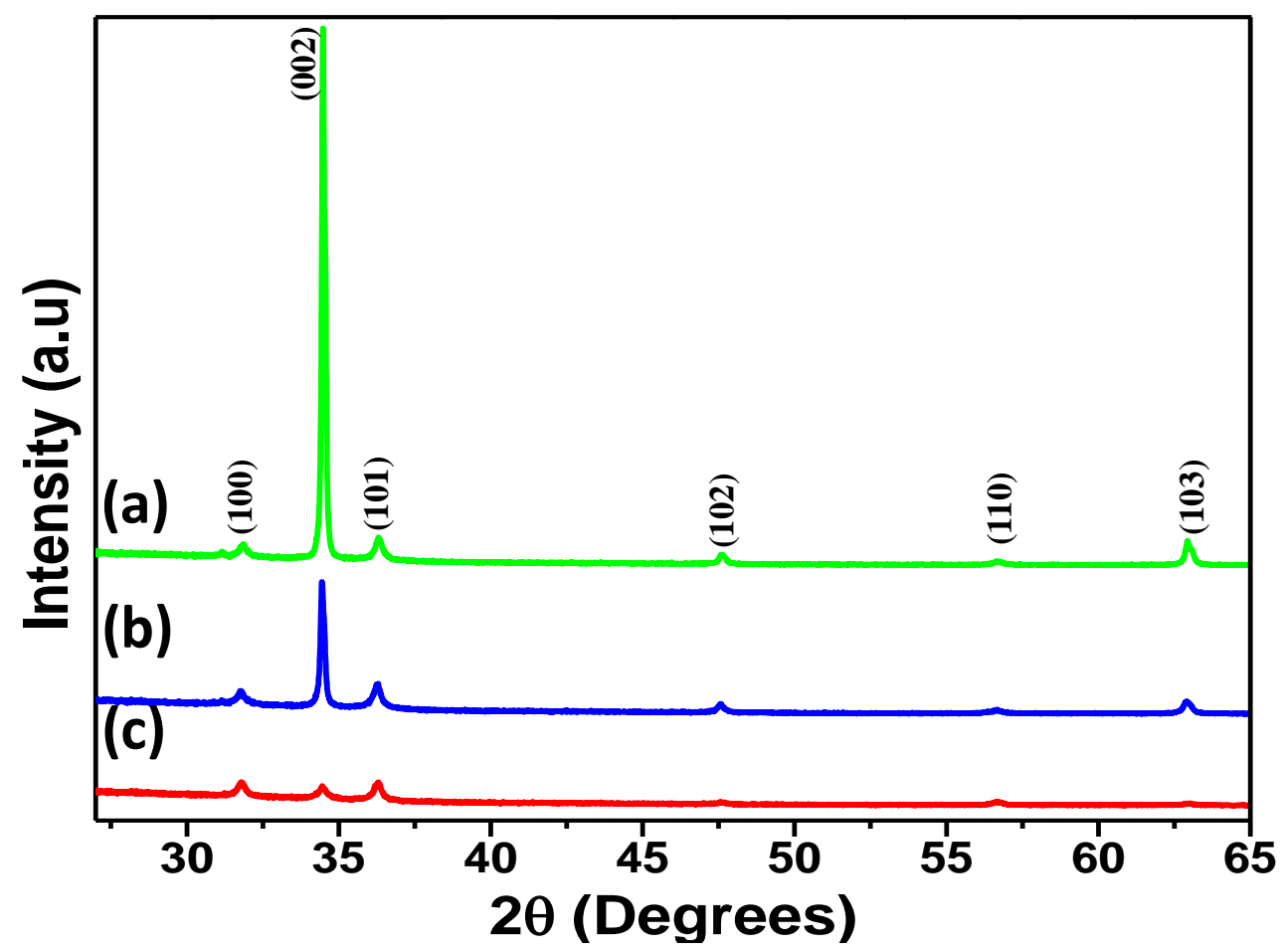

Fig. 2. XRD patterns of the as-synthesized $\mathrm{ZnO}$ nanostructures where: (a) Sample 1 (b) Sample 2 and (c) Sample 3

Table 1. Intensity and FWHM of the synthesized nanostructures extracted from the spectra of Fig. 2.

\begin{tabular}{|c|c|c|c|c|c|c|c|}
\hline \multicolumn{2}{|c|}{$\begin{array}{c}\text { JCPDS } \\
(36-1451)\end{array}$} & \multicolumn{2}{|c|}{ Sample 1} & \multicolumn{2}{|c|}{ Sample 2} & \multicolumn{2}{|c|}{ Sample 3} \\
\hline$h k l$ & Intensity & Intensity & $F W H M$ & Intensity & $F W H M$ & Intensity & $F W H M$ \\
\hline (100) & 57 & 6 & 0.146 & 13 & 0.184 & 27 & 0.208 \\
\hline (002) & 44 & 100 & 0.114 & 100 & 0.155 & 22 & 0.210 \\
\hline (101) & 100 & 14 & 0.153 & 42 & 0.168 & 100 & 0.148 \\
\hline
\end{tabular}

Table 1 shows XRD data of the synthesized nanostructures and compares the intensities of the synthesized nanostructures with the JCPDS data file $36-1451$. The full width at half 
maximum (FWHM) of all three samples were less than $0.25^{\circ}$ indicating the highly crystalline nature of the nanostructures. When comparing the relative intensities of each morphology with the JCPDS data, the preferred orientational growth of Sample 1 perpendicular to the surface is prominently shown by the high intensity of the (002) peak. This is much less in Sample 2, and in Sample 3 the ratios of the relative peak intensities are much closer to the randomly orientated powder diffraction data. The average crystallite sizes, $D(\mathrm{~nm})$ calculated using the Debye-Scherrer's equation [10] was $64.8 \mathrm{~nm}, 50.7 \mathrm{~nm}$ and $46.0 \mathrm{~nm}$ for Sample 1, Sample 2 and Sample 3, respectively.

\subsection{Optical Properties}

\subsubsection{UV-Vis Absorption}

Fig. 3 presents the RT optical absorption spectra of the different morphologies of the synthesized $\mathrm{ZnO}$ nanostructures. Normally, the optical absorption of nanostructures is assigned to the electronic transitions regarding the conduction band, the valence band and various intrinsic defect levels [11]. The spectra shows absorption peaks at $379 \mathrm{~nm}, 382 \mathrm{~nm}$ and $384 \mathrm{~nm}$ for Sample 1, Sample 2 and Sample 3, respectively. It is clearly observed that the absorption peaks showed a red shift as the crystallite size of the samples decreases. The crystallite sizes of the nanostructures found to decrease from Sample 1 to Sample 3 as discussed in section 3.2. This shift has previously been attributed to the quantum size effects as explained in quantum confinement theory $[12,13]$. 


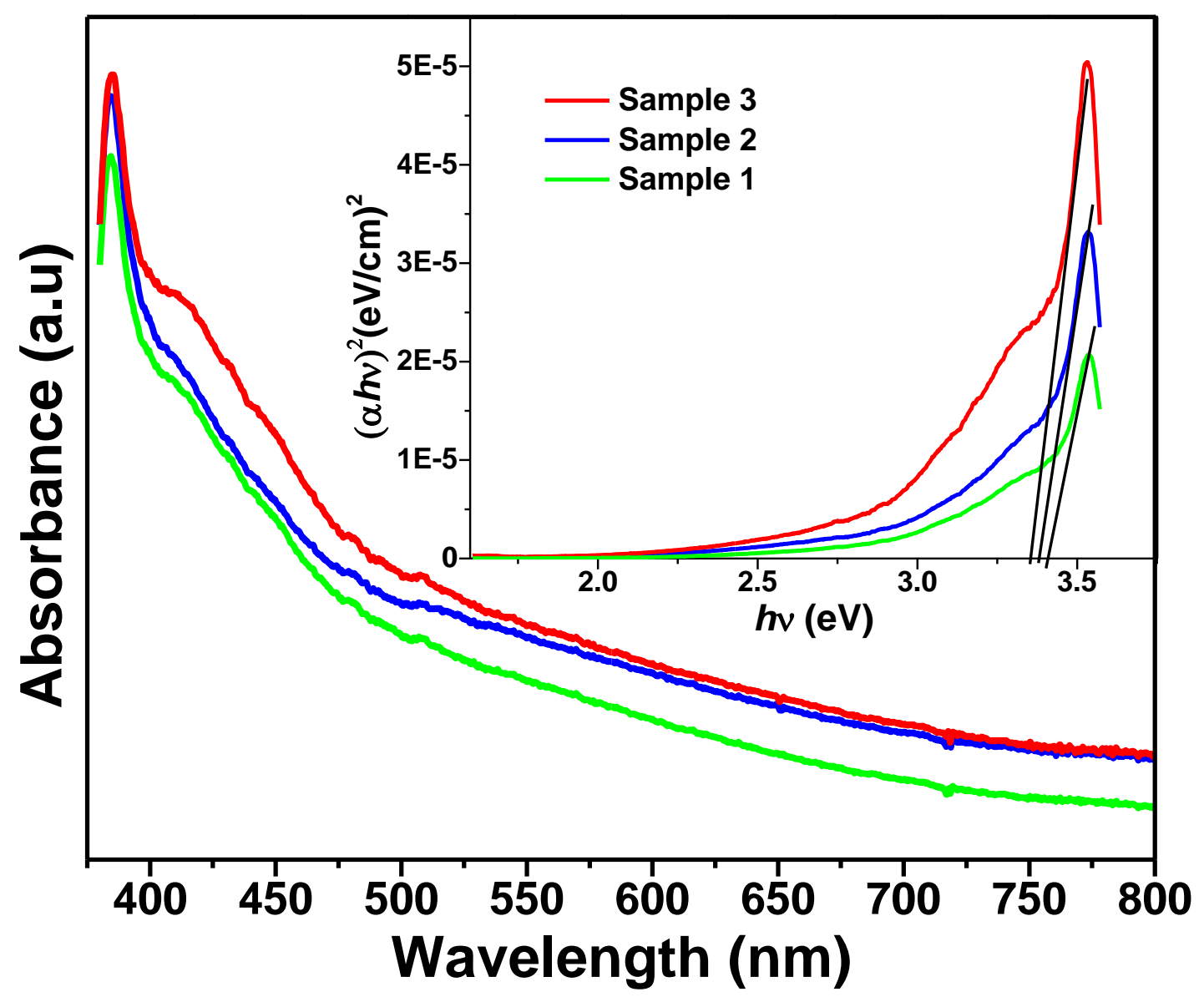

Fig. 3. Room temperature absorption spectra of different morphologies of $\mathrm{ZnO}$ nanostructures. The insert shows Tauc plot for extrapolation of optical band gap.

The optical band gaps of the three samples were studied using RT optical absorbance using Tauc equation as reported by [14]. The optical band gap was evaluated from the plot of $(\alpha h v)^{2}$ versus $h v(\mathrm{eV})$ as shown in the insert of Fig. 3. The absorption coefficient $\alpha$, were calculated using $\alpha=2.303 \log (A / d)$ (A is the absorbance and $d$ is the thickness/length of the nanostructures as measured from cross section SEM images). The optical band gap energies of Sample 1, Sample 2 and Sample 3 were $3.38 \mathrm{eV}, 3.36 \mathrm{eV}$ and $3.34 \mathrm{eV}$, respectively and were in accordance with the crystallite size variation estimated from XRD spectra. This result was in good agreement with the study reported elsewhere [15] that decreased optical band gap was attributed to the changes in surface morphologies and particle sizes. Generally, the 
optical band gaps of these nanostructures were in good agreement with the values previously reported [16].

\subsubsection{Photoluminescence}

The optical properties of the synthesized $\mathrm{ZnO}$ nanostructures were further investigated by PL. $\mathrm{ZnO}$ exhibits two kind of emission: the first one is the near band edge (NBE) emission which is due to the recombination of free excitons emission of $\mathrm{ZnO}$ [10]. The second comprises one or more visible emission corresponding to deep levels (DL) with peaks ranging from $\sim 400$ $\mathrm{nm}$ to $\sim 730 \mathrm{~nm}$ which are related to various impurities and structural defects (oxygen vacancies and zinc interstitials) [17]. Significant exciton band edge emissions in the ultraviolet (UV) region were observed irrespective of the differences in the surface morphologies of the nanostructures as presented in Fig. 4. The weak emission peaks observed in the wavelength ranging from $472 \mathrm{~nm}$ to $499 \mathrm{~nm}$ (as shown in an insert of Fig. 4) corresponds to blue color emission which was reported to originate from zinc interstitials defects in $\mathrm{ZnO}[18]$. 


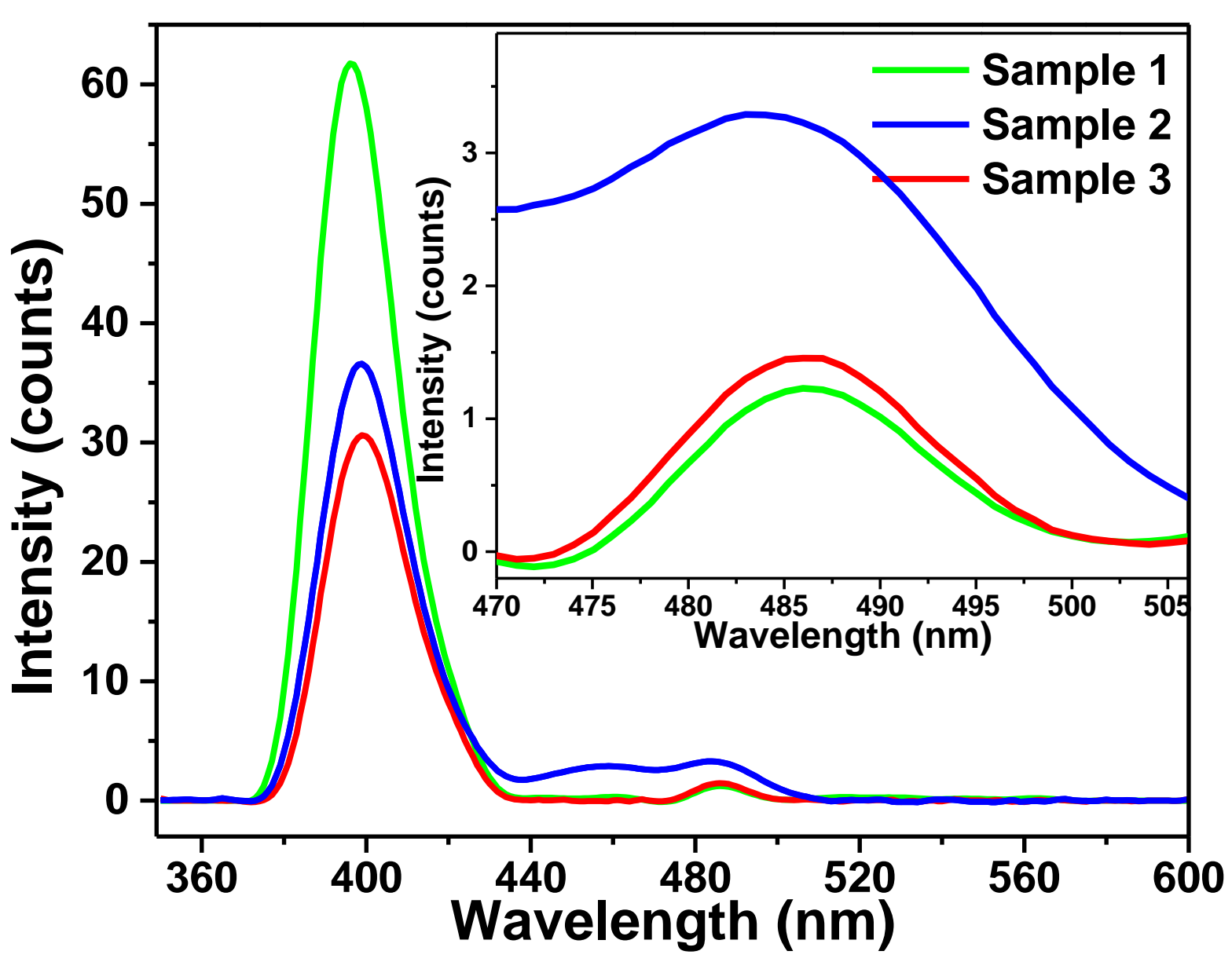

Fig. 4 Room temperature PL spectroscopy of $\mathrm{ZnO}$ nanostructures. The insert is the magnified DLE peak.

\subsection{Raman Studies}

Room temperature Raman spectra for the three different morphologies of $\mathrm{ZnO}$ nanostructures are displayed in Fig. 5. Wurzite structure of $\mathrm{ZnO}$ can results in twelve phonon branches, nine optical and three acoustic, as described elsewhere [19]. The observed characteristic Raman frequencies are the $E_{2}$ (low) at $99 \mathrm{~cm}^{-1}, \mathrm{E}_{2}$ (high) at $439 \mathrm{~cm}^{-1}$, $\mathrm{E}_{2}$ (high) - $\mathrm{E}_{2}$ (low) at $335 \mathrm{~cm}^{-1}, \mathrm{~A}_{1}$ longitudinal optical (LO) at $571 \mathrm{~cm}^{-1}$ and $\mathrm{E}_{1}(\mathrm{LO}) 585 \mathrm{~cm}^{-1}$ modes. 


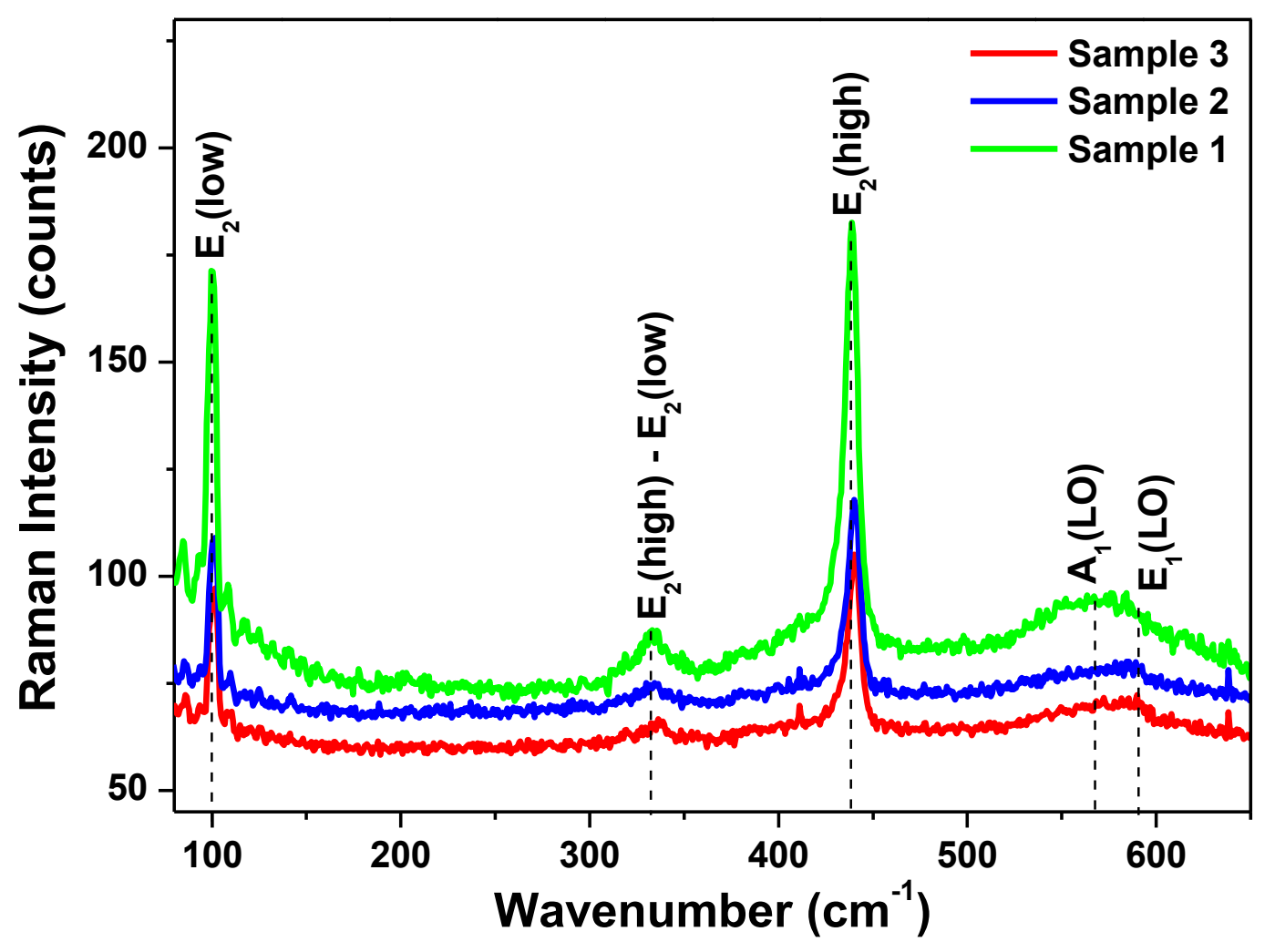

Fig. 5 Room temperature Raman spectra of $\mathrm{ZnO}$ nanostructures for the three different samples.

The $\mathrm{E}_{2}$ (high) mode is related to the oxygen atoms and $\mathrm{E}_{2}$ (low) mode is associated to the vibration of heavy zinc sub lattices. The synthesized nanostructures were all crystalline as indicated by sharp $E_{2}$ (high) phonon peak which is the significant feature for the wurtzite lattice structure of hexagonal $\mathrm{ZnO}$. However, the intensities of $\mathrm{E}_{2}$ (low) and $\mathrm{E}_{2}$ (high) peaks of Sample 1 displays the highest intensities as compared to Sample 2 and 3 indicating high degree of crystallinity of the well-aligned nanorods. This agrees well with the XRD results presented in Section 3.2. $\mathrm{E}_{1}(\mathrm{LO})$ peak were previously reported as due to defects present in $\mathrm{ZnO}$ due to doping and/or other post treatment of the sample [20,21]. In the present study, we eliminate the idea that the peak at $582 \mathrm{~cm}^{-1}$ was due to these defects which concurs with results reported for both undoped and doped $\mathrm{ZnO}$ nanorods [22]. However, further systematic studies on Raman defects related peaks by using are needed for as-synthesized CBD ZnO nanostructures. Another important feature is the shift to higher frequencies in the $\mathrm{E}_{2}(\mathrm{low})$, $E_{2}$ (high) and $E_{2}$ (high) $-E_{2}$ (low) Raman peaks of the rod-like (Sample 2) and agglomerated 
nanoparticles (Sample 3). The shift could be due to spatial confinement, phonon localization and defects which are more pronounced for chemically synthesized nanostructures [23].

\subsection{Electrical Characterization}

The effect of morphologies on the electrical properties was comparatively studied. Fig. 6 shows linear plot of $I-V$ characteristics of Schottky diodes made by evaporating Au metal on the samples. They all displayed non-linear rectifying behavior of Schottky diode with a turnon voltage of $0.39 \mathrm{~V}$ for Sample 1, $0.43 \mathrm{~V}$ for Sample 2 and $0.55 \mathrm{~V}$ for Sample 3. The insert of Fig. 6 clearly demonstrates ohmic characteristics of the ITO substrate, as it displays linear $I-V$ characteristic for both reverse and forward bias.

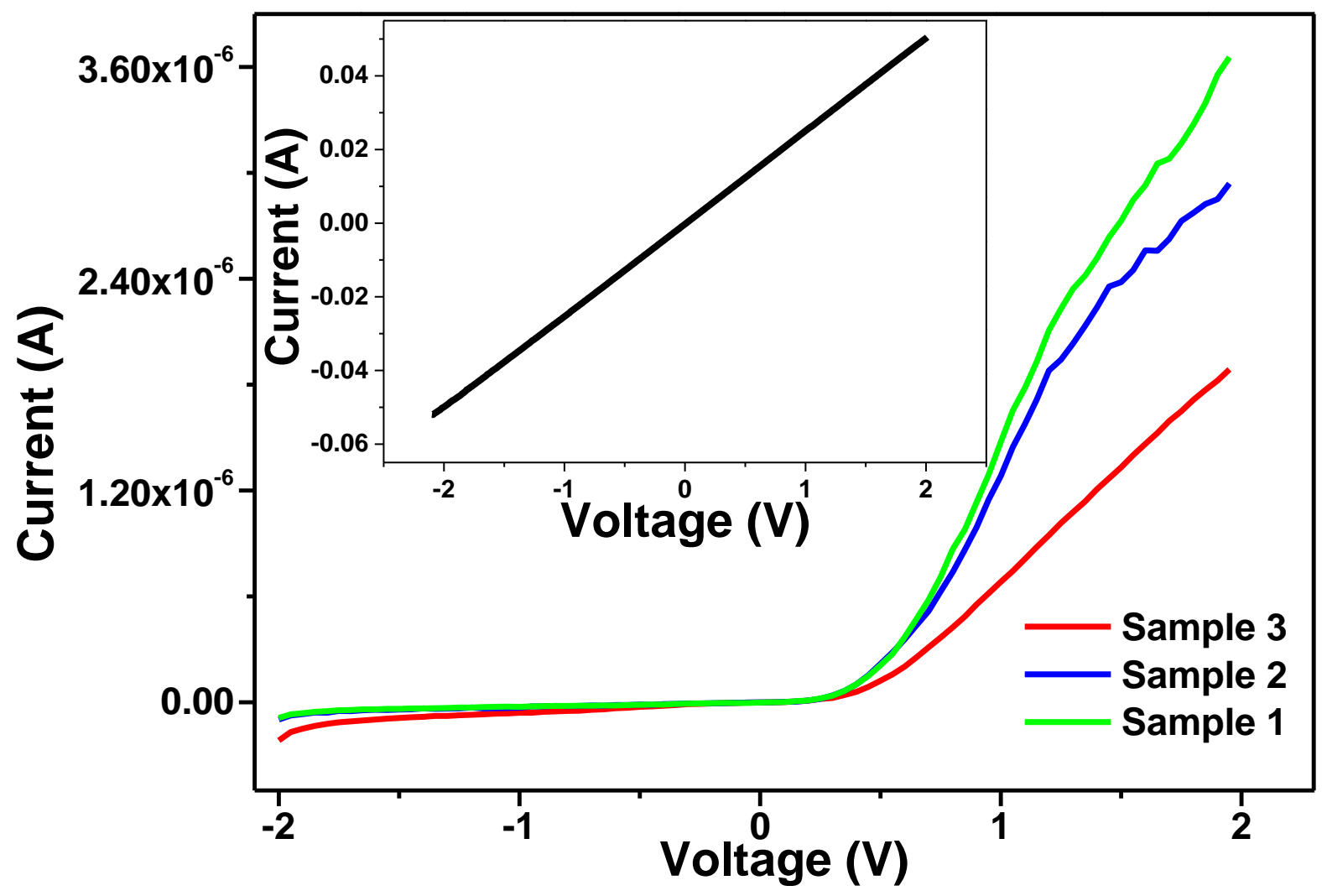

Fig. 6. $I-V$ characteristics of Au/ZnO/ITO Schottky diode in the dark. The insert graph shows linear $I-V$ characteristics of ITO ohmic contact.

Fig. 7 shows semi-logarithmic $I-V$ characteristics as the function of bias voltage. The forward current was higher for hexagonally shaped $\mathrm{ZnO}$ nanorods in Sample 1 as compared to the 
other structures. It was further observed that at lower voltages $0.1-0.3 \mathrm{~V}$ forward currents increase exponentially, and at higher voltages $0.5-2.0 \mathrm{~V}$, approximately linearly. From this result, it is evident that $\mathrm{Au}$ metal was successfully deposited on the nanostructures forming metal-semiconductor (M/S) junction with significant series resistance. Insert of Fig. 7 displays the rectification ratio which was obtained by dividing the forward current $\left(I_{\mathrm{F}}\right)$ and reverse current $\left(I_{\mathrm{R}}\right)$ at in a forward bias voltage $\left(I_{F} / I_{R}\right)_{V}$. The low values of rectification ratio obtained could be attributed to the fact that the metal contacts were evaporated on the surface of $\mathrm{ZnO}$ nanostructures without cleaning. According to Mtangi et al [24], good rectifying $\mathrm{ZnO}$ Schottky diodes have been difficult to achieve due the difficulties in obtaining a clean surface. However, higher rectification ratio has been obtained with vertically aligned nanorods compared to the other two structures. This agrees well with literature that highly oriented nanostructures demonstrates good performance [25].

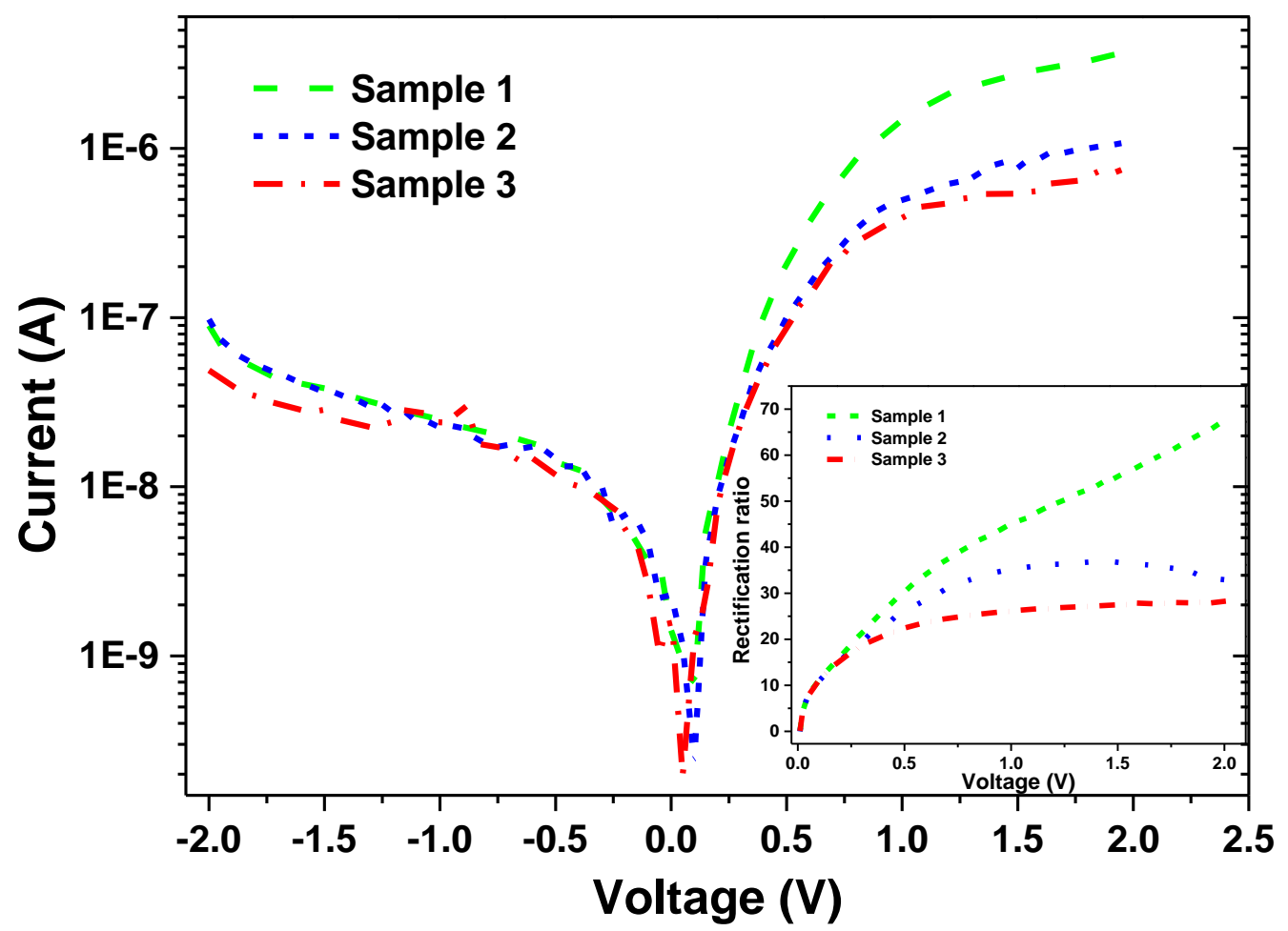

Fig. 7. Semi logarithmic $I-V$ characteristics of Au/ZnO/ITO Schottky diode in the dark. The insert graph shows rectification ratio dependence on the applied bias for the three morphological structures. 
Values of the Schottky barrier height $\Phi_{B O}$, ideality factor $n$, series resistance $R_{S}$ and reverse saturation current $I_{0}$ of the three structures were calculated from the $I-V$ measurement based on standard Thermionic emission theory of the Schottky diode as described by Sze [26] and presented in Table 2. From the table, $n$ values decreased from 1.96 to 1.73 and 1.57 whereas $\Phi_{B 0}$ increased from 0.81 to $0.90 \mathrm{eV}$ and $0.93 \mathrm{eV}$, for Sample 1 to Sample 2 and Sample 3, respectively. All samples present $n$ greater than 1 indicating the non-ideal behavior of the fabricated Schottky diodes and could be attributed to among others, inhomogeneities of the top surface of the nanostructures (as seen in Fig. 1 b - d), series resistance, nonuniform distribution of the interfacial charges and voltage drop across the M/S junction [5]. According to the Schottky-Mott theory, the expected $\Phi_{B O}$ for $\mathrm{Au} / \mathrm{ZnO}$ nanostructures/ITO is $\sim 0.8 \mathrm{eV}$ the difference between the $\mathrm{Au}$ work-function and the $\mathrm{ZnO}$ nanorods electron affinity of $4.27 \mathrm{eV}$. For these nanostructures, the $\Phi_{B 0}$ were measured $0.81 \mathrm{eV}$ (Sample 1) and slightly higher values of $0.90 \mathrm{eV}$ and $0.93 \mathrm{eV}$ for Sample 2 and Sample 3, respectively. The $\Phi_{B 0}$ deviation have been previously attributed to the non-homogeneous barrier formation, Schottky effect (image-force-induced lowering) and dark regions that do not contribute to the current transport over the Schottky barrier [27,28]. The observed large series resistance for Sample 2 and Sample 3 as listed in the Table 2 agrees well with the semi-log plot of Fig. 7 where a considerable deviation from linearity is more pronounced indicating increased series resistance. In the present study, $I-V$ parameters were largely affected by the surface morphologies of the nanostructures. As observed Sample 1 demonstrated good electrical properties (lowest $R_{S}$, highest rectification ratio and good $\Phi_{B 0}$, close to theoretical value) compared to Sample 2 and Sample 3. This is because vertically aligned nanorods (Sample 1), gave an advantage of higher vertical conduction as they use one dimensional transport in a direction perpendicular to the conducting substrate, which was difficult to achieve for nanorods lying horizontally and the densely agglomerated nanoparticles. 
Table 2. Electrical parameters of $\mathrm{Au} / \mathrm{ZnO}$ nanostructures/ITO Schottky diode.

\begin{tabular}{lcccc}
\hline & Ideality factor $(n)$ & $\begin{array}{c}\text { Barrier height } \\
\left(\Phi_{B 0}, \mathrm{eV}\right)\end{array}$ & Series resistance $(\Omega)$ & $\begin{array}{c}\text { Reverse saturation } \\
\text { current }\left(I_{0}, \mathrm{~A}\right)\end{array}$ \\
\hline Sample 1 & 1.96 & 0.81 & 655.3 & $4.91 \times 10^{-6}$ \\
Sample 2 & 1.57 & 0.90 & 2948.6 & $5.99 \times 10^{-7}$ \\
Sample 3 & 1.57 & 0.93 & 3352.2 & $2.58 \times 10^{-7}$ \\
\hline \hline
\end{tabular}

It was also reported that Schottky diodes based on $\mathrm{ZnO}$ nanostructures/thin films synthesized using chemical methods in air can induce interfacial dielectric layers that significantly alter Schottky barrier heights and strongly affect the current of the diode [27]. Moreover, Aydoğan et al [29], reported that large values of $\Phi_{B 0}$ may be attributed to the electronic states that may cause a reduction or increase in the metal work function at the interface resulting in an increase or decrease in the barrier height.

The transport mechanisms in our fabricated diodes were studied by using double -logarithmic $I-V$ characteristics of the forward bias as shown in Fig. 8. Power law $I=k V^{m}$ was used where $I$ is the current, $k$ is a constant, $V$ is the voltage and $m$ is a constant describing charge transport mechanism which is equal to the slope of the $\log I-\log V$ graph. As seen in Fig. 7, the plot shows two linear regions, region 1 and region 2 . The $m$ values for region 1 are 2.99 , 2.85 and 3.09, for Sample 1, Sample 2 and Sample 3, respectively and for region 2 were found to be 2.19, 2.68 and 2.46 for Sample 1, Sample 2 and Sample 3, respectively. From the results, it is clear that both regions I and II follows power law $\left(I \approx V^{m}\right)$, suggesting that space charge limited current (SCLC) is the dominant transport mechanism [30]. However, we see that the slopes at region 2 for all samples were slightly reduced as compared to those of region 1 . This could be due to the fact that device is approaching the so called trap-filled limit as described elsewhere [29,31,32]. 


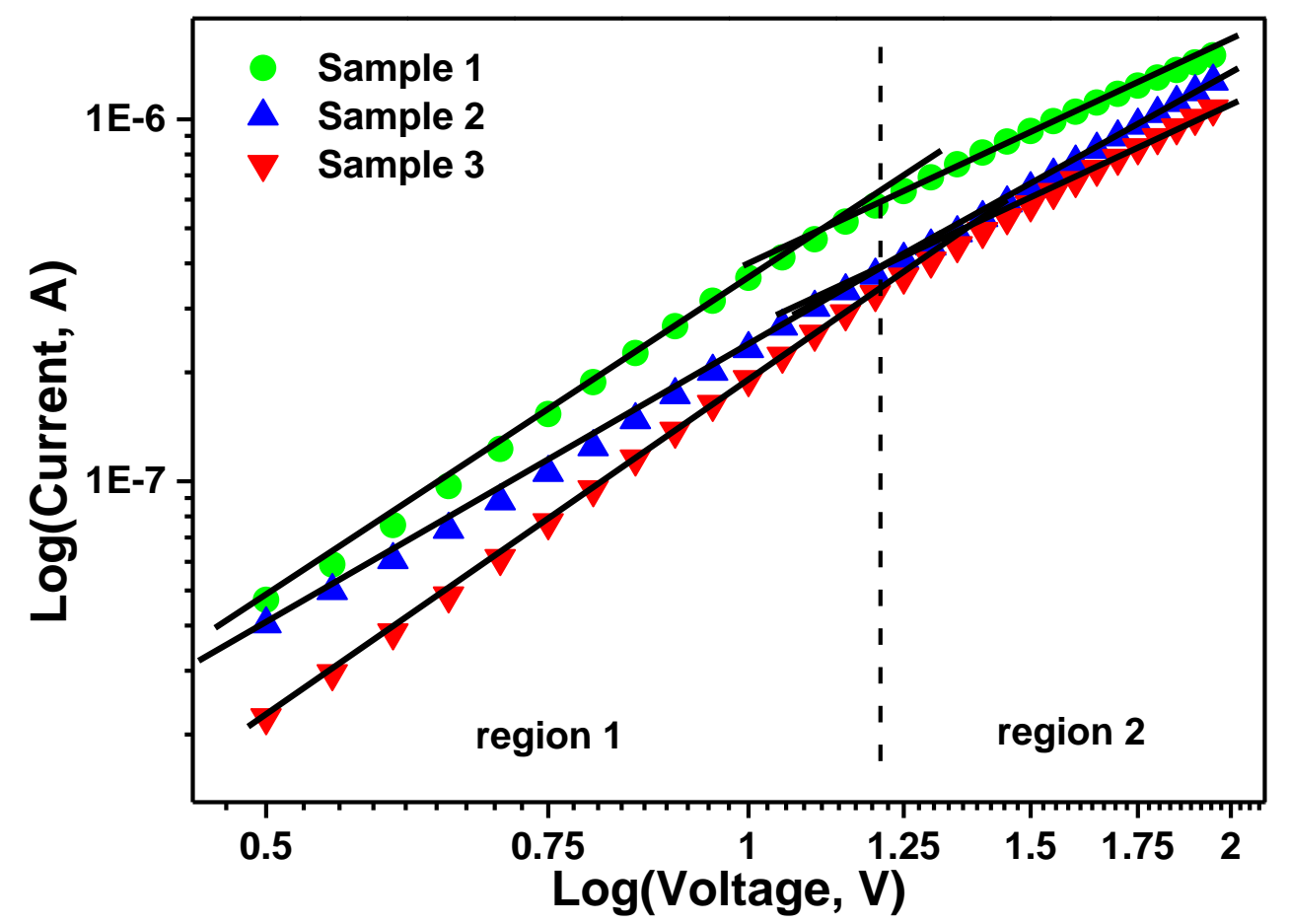

Fig. 8. $I-V$ characteristics in a double-logarithmic plot for $\mathrm{Au} / \mathrm{ZnO} / \mathrm{ITO}$ Schottky diode in the higher voltage range of the forward bias.

\section{Conclusion}

$\mathrm{ZnO}$ nanostructures were successfully synthesized using surfactant and catalyst free CBD methods. The FESEM images revealed three different morphologies of nanostructures: 1) vertically aligned hexagonal nanorods having flat tops, 2) narrower nanorods lying parallel to the substrate, and 3) randomly oriented agglomerated nanoparticles and these resulted when the seeded substrate was placed facing down, vertically and up in the beaker, respectively. The optical absorption band edge was blue shifted as compared to the bulk and optical band gap energy increased both for Sample 1 and Sample 2 increased while for Sample 3 this decreased. Raman studies show sharp and strong $\mathrm{E}_{2}$ (high) phonon peak which is a significant feature for the wurtzite lattice structure of hexagonal $\mathrm{ZnO}$. The $I-V$ characteristics display non-linear rectifying behavior of the Schottky diode with a turn-on voltage of $0.39 \mathrm{~V}, 0.43 \mathrm{~V}$ and 0.55 V for Sample 1, Sample 2 and Sample 3, respectively. Vertically aligned ZnO nanorods (Sample 1) display the highest rectification ratio of 69 at $\pm 2 \mathrm{~V}$ as compared to 33 
and 27 at $\pm 2 \mathrm{~V}$ for Sample 2 and Sample 3, respectively. The Schottky diode parameters including ideality factor, barrier height and series resistance have been calculated and found to depend on the morphology of the synthesized nanostructures. Sample 1 demonstrates a $\Phi_{B O}$ of $0.81 \mathrm{eV}$ which is almost equal to the theoretical value $(0.8 \mathrm{eV})$, while Sample 2 and Sample 3 show higher $\Phi_{B 0}$ values of 0.90 and $0.93 \mathrm{eV}$, respectively. According to power law, the space charge limited current (SCLC) was found to be the dominant transport mechanism in the higher voltage range of the forward bias.

\section{Acknowledgements}

The work was financially supported by the University of Pretoria and National Research Foundation (NRF), South Africa, Grant No: 91550 and 94166.

\section{References}

[1] D.H. Kim, J.H. Park, T. Il Lee, J.M. Myoung, Superhydrophobic Al-doped ZnO nanorods-based electrically conductive and self-cleanable antireflecting window layer for thin film solar cell, Sol. Energy Mater. Sol. Cells. 150 (2016) 65-70. doi:10.1016/j.solmat.2016.01.041.

[2] K.L. Foo, U. Hashim, K. Muhammad, C.H. Voon, Sol - gel synthesized zinc oxide nanorods and their structural and optical investigation for optoelectronic application, 9 (2014) 1-10. doi:10.1186/1556-276X-9-429.

[3] M. Abd-Ellah, J.P. Thomas, L. Zhang, K.T. Leung, Enhancement of solar cell performance of p-Cu2O/n-ZnO-nanotube and nanorod heterojunction devices, Sol. Energy Mater. Sol. Cells. 152 (2016) 87-93. doi:10.1016/j.solmat.2016.03.022.

[4] M.Y.A. Rahman, A.A. Umar, R. Taslim, M.M. Salleh, Effect of surfactant on the physical properties of $\mathrm{ZnO}$ nanorods and the performance of $\mathrm{ZnO}$ photoelectrochemical cell, J. Exp. Nanosci. 10 (2015) 599-609. 
doi:10.1080/17458080.2013.858374.

[5] N.S. Singh, L. Kumar, A. Kumar, S. Vaisakh, S.D. Singh, K. Sisodiya, S. Srivastava, M. Kansal, S. Rawat, T.A. Singh, Tanya, Anita, Fabrication of zinc oxide/ polyaniline ( $\mathrm{ZnO} / \mathrm{PANI})$ heterojunction and its characterisation at room temperature, Mater. Sci. Semicond. Process. 60 (2017) 29-33. doi:10.1016/j.mssp.2016.12.021.

[6] Z. Liang, Q. Zhang, O. Wiranwetchayan, J. Xi, Z. Yang, K. Park, C. Li, G. Cao, Effects of the morphology of a $\mathrm{ZnO}$ buffer layer on the photovoltaic performance of inverted polymer solar cells, Adv. Funct. Mater. 22 (2012) 2194-2201. doi:10.1002/adfm.201101915.

[7] S. Oh, S. Kim, S.-C. Shin, P.-C. Su, Y.-J. Yoon, K.J. Lee, S.-J. Kim, H. Choi, S.-J. Park, K.-K. Kim, Morphology Effect of the ZnO Surface via Organic Etchants for Photon Extraction in III-Nitride Emitters, ECS J. Solid State Sci. Technol. 6 (2017) Q13-Q17. doi:10.1149/2.0131701jss.

[8] B.S. Mwankemwa, M.J. Legodi, M. Mlambo, J.M. Nel, M. Diale, Structural, Morphological, Optical and Electrical properties of Schottky diodes based on CBD deposited ZnO:Cu Nanorods, Superlattices Microstruct. 107 (2017) 163-171. doi:10.1016/j.spmi.2017.04.018.

[9] A. Resmini, I.G. Tredici, C. Cantalini, L. Giancaterini, F. De Angelis, E. Rondanina, A simple all-solution approach to the synthesis of large $\mathrm{ZnO}$ nanorod networks, J. Mater. Chem. A Mater. Energy Sustain. 3 (2015) 4568-4577. doi:10.1039/C4TA05207B.

[10] T.K. Pathak, A. Kumar, C.W. Swart, H.C. Swart, R.E. Kroon, Effect of fuel content on luminescence and antibacterial properties of zinc oxide nanocrystalline powders synthesized by the combustion method $\uparrow$, RSC Adv. 6 (2016) 97770-97782. doi:10.1039/C6RA22341A.

[11] Q. Zhu, C. Xie, H. Li, C. Yang, S. Zhang, D. Zeng, Selectively enhanced UV and NIR 
photoluminescence from a degenerate $\mathrm{ZnO}$ nanorod array film, J. Mater. Chem. C. 2 (2014) 4566-4580. doi:10.1039/c4tc00011k.

[12] N.S. Pesika, K.J. Stebe, P.C. Searson, Relationship between Absorbance Spectra and Particle Size Distributions for Quantum-Sized Nanocrystals, J. Phys. Chem. B. 107 (2003) 10412-10415. doi:10.1021/jp0303218.

[13] M.D.R. Balaguera-Gelves, Improved Low-Temperature Aqueous Synthesis of ZnO Nanorods and Their Use in SERS Detection of 4-ABT and RDX, Mater. Sci. Appl. 4 (2013) 29-38. doi:10.4236/msa.2013.41005.

[14] H.S. Al-Salman, M.J. Abdullah, Structural, optical, and electrical properties of Schottky diodes based on undoped and cobalt-doped $\mathrm{ZnO}$ nanorods prepared by RFmagnetron sputtering, Mater. Sci. Eng. B Solid-State Mater. Adv. Technol. 178 (2013) 1048-1056. doi:10.1016/j.mseb.2013.06.011.

[15] M.A. Desai, S.D. Sartale, Facile soft solution route to engineer hierarchical morphologies of ZnO nanostructures, Cryst. Growth Des. 15 (2015) 4813-4820. doi:10.1021/acs.cgd.5b00561.

[16] F. Ahmed, S. Kumar, N. Arshi, M.S. Anwar, R. Prakash, Growth and characterization of $\mathrm{ZnO}$ nanorods by microwave-assisted route : green chemistry approach, 2 (2011) 183-187. doi:10.5185/amlett.2011.1213.

[17] N. Tripathi, S. Rath, Facile synthesis of $\mathrm{ZnO}$ nanostructures and investigation of structural and optical properties, Mater. Charact. 86 (2013) 263-269. doi:10.1016/j.matchar.2013.10.008.

[18] C. Singh, E. Panda, Variation of electrical properties in thickening Al-doped ZnO films : Role of defect chemistry RSC Advances Variation of electrical properties in thickening, RSC Adv. 6 (2016) 48910-48918. doi:10.1039/C6RA06513A.

[19] R. Das, A. Kumar, Y. Kumar, Effect of growth temperature on the optical properties of 
ZnO nanostructures grown by simple hydrothermal method, RSC Adv. 5 (2015) 60365-60372. doi:10.1039/C5RA07135F.

[20] A. Al-na, B. Sieber, B. Gelloz, A. Addad, M. Moreau, J. Barjon, M. Girleanu, O. Ersen, R. Boukherroub, Enhanced Ultraviolet Luminescence of $\mathrm{ZnO}$ Nanorods Treated by High-Pressure Water Vapor Annealing ( HWA ), J. Physi. Chem. C. 120 (2016) 4571-4580. doi:10.1021/acs.jpcc.5b09201.

[21] M.A. Gaikwad, M.P. Suryawanshi, S.S. Nikam, C.H. Bhosale, J.H. Kim, A. V Moholkar, Influence of $\mathrm{Zn}$ concentration and dye adsorption time on the photovoltaic performance of M-SILAR deposited ZnO-based dye sensitized solar cells, "Journal Photochem. Photobiol. A Chem. 329 (2016) 246-254. doi:10.1016/j.jphotochem.2016.07.006.

[22] R. Das, A. Kumar, Y. Kumar, S. Sen, P.M. Shirage, Effect of growth temperature on the optical properties of $\mathrm{ZnO}$ nanostructures grown by simple hydrothermal method, RSC Adv. 5 (2015) 60365-60372. doi:10.1039/C5RA07135F.

[23] K.A. Alim, V.A. Fonoberov, M. Shamsa, A.A. Balandin, Micro-Raman investigation of optical phonons in ZnO nanocrystals, J. Appl. Phys. 97 (2005) 1-5. doi:10.1063/1.1944222.

[24] W. Mtangi, F.D. Auret, C. Nyamhere, P.J. Janse van Rensburg, A. Chawanda, M. Diale, J.M. Nel, W.E. Meyer, The dependence of barrier height on temperature for Pd Schottky contacts on ZnO, Phys. B Condens. Matter. 404 (2009) 4402-4405. doi:10.1016/j.physb.2009.09.022.

[25] L. Vayssieres, B.L. Vayssieres, Growth of Arrayed Nanorods and Nanowires of ZnO from Aqueous Solutions Growth of Arrayed Nanorods and Nanowires of $\mathrm{ZnO}$ from Aqueous Solutions, Adv. Mater. 15 (2015) 464-466. doi:10.1002/adma.200390108.

[26] S.M. Sze, Physics of Semiconductor Devices, Third edit, Wiley, New York, 1979. 
[27] L.J. Brillson, Y. Lu, ZnO Schottky barriers and Ohmic contacts, J. Appl. Phys. 109 (2011) 1-33. doi:10.1063/1.3581173.

[28] H. Von Wenckstern, E.M. Kaidashev, M. Lorenz, H. Hochmuth, G. Biehne, J. Lenzner, V. Gottschalch, R. Pickenhain, M. Grundmann, Lateral homogeneity of Schottky contacts on n-type ZnO, Appl. Phys. Lett. 84 (2004) 79-81. doi:10.1063/1.1638898.

[29] Ş. Aydoğan, I. Üncekara, A.R. Deniz, A. Türüt, Extraction of electronic parameters of Schottky diode based on an organic Indigotindisulfonate Sodium (IS), Solid State Commun. 150 (2010) 1592-1596. doi:10.1016/j.ssc.2010.05.043.

[30] A. Dey, A. Layek, A. Roychowdhury, M. Das, J. Datta, S. Middya, D. Das, P.P. Ray, Investigation of charge transport properties in less defective nanostructured $\mathrm{ZnO}$ based Schottky diode, RSC Adv. 5 (2015) 36560-36567. doi:10.1039/C4RA16828C.

[31] P. Kumar, A. Jain, M. Shukla, S. Chand, Space charge limited current in Schottky diode with single level traps, Indian J. Pure Appl. Phys. 49 (2011) 406-409.

[32] A. Kathalingam, Senthilkumar, S. Valanarasu, J.-K. Rhee, Shape-dependent electrical property of solution synthesized ZnO nanorods, Semicond. Sci. Technol. 27 (2012) 19. doi:10.1088/0268-1242/27/10/105006. 